\title{
A Check-List of the Mecoptera of the World
}

\author{
Norman D. Penny ( $\left.{ }^{*}\right)$ \\ George W. Byers ( $\left.{ }^{* *}\right)$
}

\begin{abstract}
All known species of recent Mecoptera are catalogued; reference is given to original descriptions; and known distributions are recorded. New names for two junior homonyms (Panorpa issiki $=$ Panorpa acuta issiki \& Cheng, and Panorpa banksiana = Panorpa inter rupta Banks) are included, and one new synonymy (Pa. norpa galloisi Miyake $=$ Panorpa cornigera MacLachlan) is noted.
\end{abstract}

\section{INTRODUCTION}

The insect order Mecoptera has become increasingly better known in recent years due to such publications as Schlee's (1977) bibliography of literature on the order; Kaltenbach's (1978) summary of the entire order; Byers' (1965c) study of family-level classification; Penny's (1977) revision of the family Boreidae; and faunal works by Martynova (1954, 1957. 1959) for the U.S.S.R., Cheng (1957a) for China, Byers (1965a) for Indo-China, Byers (1971b) and Londt (1972) for Africa, Riek (1954, 1973) for Australia, Rust and Byers (1976) for India, and Chau and Byers (1978) for Indonesia.

Not since Esben-Petersen's (1921) monographic revision has a compilation been available of all the species of Mecoptera. Since that time 41 per cent of known genera and 68 per cent of known species have been described. This check-list is provided as an aid to furthering comprehensive knowledge of the Mecoptera.

Two divergent trends among the faunal regions are shown in Tables 1 and 2 . One trend, found in the Australian, Nearctic and Neotropical Regions, is toward great diversity at the family and generic levels, but relatively few species. The other trend, existing in the Ethiopian, Oriental and Palearctic Regions, is toward slight diversity at the family and generic levels but great diversity at the species level. For example, in the combined Australian, Nearctic, and Neotropical areas can be found representatives of all families and of 25 of the 29 known genera of Mecoptera, but only 28 per cent of the known species. Conversely, the other three regions contain only four families and seven genera of Mecoptera, but 72 per cent of the known species. Actually, the Nearctic Region appears to be somewhat intermediate, having a large number of families and genera, but also a fairly high number of species. More study needs to be given to the reasons for this dichotomy.

TABLE 1. Distribution of taxa of Mecoptera by region

\begin{tabular}{lccc}
\hline Faunal Region & Families & Genera & Species \\
\hline Australian & 5 & 11 & 27 \\
Ethiopian & 1 & 2 & 53 \\
Nearctic & 5 & 7 & 72 \\
Neotropical & 3 & 9 & 34 \\
Oriental & 2 & 4 & 190 \\
Palearctic & 3 & 4 & 94 \\
\hline
\end{tabular}

TABLE 2. Distribution of taxa of Mecoptera found only in a region

\begin{tabular}{lccc}
\hline Faunal Region & Families & Genera & Species \\
\hline Australian & 1 & 10 & 27 \\
Ethiopian & 0 & 1 & 53 \\
Nearctic & 0 & 4 & 72 \\
Neotropical & 1 & 7 & 34 \\
Oriental & 0 & 2 & 190 \\
Palearctic & 0 & 1 & 94 \\
\hline
\end{tabular}

(") Instituto Nacional de Pesquisas da Amazônia, Manaus.

("*) Dep. of Entomology, University of Kansas, Lawrence, U.S.A. 
Another observation is the relative scarcity of Mecoptera on islands. No Mecoptera have been found on Madagascar, although several species are recorded from nearby parts of Africa. In the South Pacific, only one endemic species exists in New Zealand. In the Caribbean, Mecoptera are known only from Trinidad. In most island areas where Mecoptera are known (i.e., Japan, Taiwan, Sri Lanka (Ceylon), Borneo, Java, Sumatra, Tioman Island, Trinidad and St. Paul Island) (") there is a geological history of relatively recent land connection to the adjacent continent. This suggests low vagility in the Mecoptera.

In the following list, when a scientific name is followed by two authors' names, the first indicates original describer, and the second the name of the author who first changed the generic status of the species. Valid subspecific names follow the specific names as non-indented trinominals, while synonymized subspecies are indented. Varieties have been omitted. This list includes names received up to 1 December 1978. The name Esben-Petersen has been abbreviated as E.P.

Two species are recorded as previously unresolved junior homonyms, and they have been given new names. The first, Panorpa issiki, was originally named Panorpa acuta by Issiki and Cheng in 1947. However, this name is predated by Panorpa acuta Carpenter (1931). The second junior homonym was originally described as Panorpa interrupta by Banks in 1918. However, in 1913 Longinos Navás described Aulops interrupta, which was later synonymized with Panorpa japonica. As the generic name Aulops has also been synonymized with Panorpa, if the species name Aulops interrupta is resurrected, it would be called Panorpa interrupta. Thus, the need to change the name proposed by Banks. We have named this species Panorpa banksiana in honor of its original describer.

One new synonomy is also included. Panorpa galloisi Navás (1913) has long been considered a synonym of Panorpa cornigera MacLachlan (1887). One pale variety of Panorpa galloisi Navás was collected at the same place and day as the type of Panorpa galloisi Miyake (1911). Based on published descriptions, noted variation between Panorpa galloisi Miyake and Panorpa galloisi Navás can be interpreted as intraspecific variation. and thus both Panorpa galloisi Miyake and and Panorpa galloisi Navás are synonyms of $\mathrm{Pa}$ norpa cornigera MacLachlan.

\section{Family APTEROPANORPIDAE}

APTEROPANORPA Carpenter (1941)

Apteropanorpa tasmanica Carpenter (1941)

Family BITTACIDAE

ANABITTACUS Kimmins (1929)

Anabittacus iridipennis Kimmins (1929)

ANOMALOBITTACUS Kimmins (1928)

Anomalobittacus gracilipes Kimmins (1928)

APTEROBITTACUS MacLachlan (1893)

Apterobittacus apterus (MacLachlan) MacLachlan (1893)

Bittacus apterus MacLachlan (1871)

AUSTROBITTACUS Riek (1954)

Austrobittacus anomalus Riek (1954)
Chile

Tasmania

\section{South Africa}

U.S.A.

Australia

(*) The Snow Entomological Museum has a specimen of Bittacus from Socotra Island which apparently does not fit in this category. 


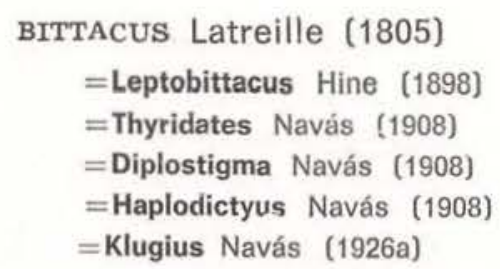

Bittacus aequalis Navás (1914)

Bittacus africanus E.-P. (1915)

Bittacus alluaudi Navás (1914)

=Bittacus jeanneli Navás (1914)

= Bittacus zavattarii Capra (1939)

Bittacus andinus Londt \& Byers (1974)

Bittacus angrensis Souza Lopes \& Mangabeira (1942)

Bittacus annae Londt (1972)

Bittacus apicalis Hagen (1861)

$=$ Haplodictyus apicalis (Hagen) Navás (1908)

$=$ Haplodictyus incertus Navás (1926b)

Bittacus appendiculatus E.-P. (1927)

Bittacus armatus Tjeder (1956b)

Bittacus banksi E.-P. (1915)

Bittacus berlandi Capra (1939)

$=$ Bittacus rhombicus Kimmins (1957)

Bittacus boraceiensis Morgante (1967)

Bittacus boranicus Capra (1939)

Bittacus brasiliensis Klug (1838)

Bittacus brunneus E.-P. (1927)

Bittacus bullatus Londt (1972)

Bittacus burgeoni Navás (1930b)

Bittacus burmanus Tjeder (1974)

Bittacus byersi Londt (1972)

Bittacus capensis (Thunberg) Klug (1838)

$=$ Panorpa capensis Thunberg (1784)

Bittacus caprai Londt (1972)

Bittacus carpenteri Cheng (1957a)

Bittacus chevalieri (Navás) E.-P. (1921)

$=$ Haplodictyus chevalieri Navás (1908)

Bittacus chilensis Klug (1838) $=$ Thyridates chilensis (Klug) Navás (1908)

Bittacus chlorostigma MacLachlan (1881)

Bittacus chujoi Issiki \& Cheng (1947)

Bittacus cirratus Tjeder (1956a)

= Bittacus zoensis Cheng (1957a)

Bittacus contumax Tjeder (1956a)

Bittacus coreanus Issiki (1929b)

Bittacus cottrelli Londt (1972)

Bittacus discors Navás (1914)

=Bittacus patrizii Navés (1929)

Bittacus diversinervis Souza Lopes \& Mangabeira (1942)

Bittacus elisabethae Navás (1930b)

Bittacus erythrostigma Byers (1975b)

Bittacus femoralis Klug (1838) eastern Africa

western Africa

eastern Africa

Bolivia, Peru

Brazil

South Africa

U.S.A.

China

Rhodesia, South Africa

Mexico to Panama

Tanzania

Brazil

E. Africa

Argentina, Brazil

Paraguay

South Africa

Zaire

Burma

South Africa

South Africa

Rhodesia, Zambia

China

western Africa

Chile

western U.S.A.

Taiwan

Manchuria

China

China, Korea

South Africa

eastern Africa

Brazil

Zaïre

Uganda, Zaïre

Brazil 
Bittacus flavescens Klug (1838)

Brazil

$=$ Kiugius flavescens (Klug) Navás (1926a)

$=$ Bittacus affinis Westwood (1846)

Bittacus formosanus Issiki (1927)

Bittacus fumosus E.-P. (1913b)

Bittacus geniculatus Erichson (1848)

Bittacus gessi Londt (1972)

Bittacus gressitti Cheng (1957a)

Bittacus hageni Brauer (1860)

Bittacus henryi Kimmins (1928)

Bittacus homburgerae Navás (1933a)

Bittacus indicus Walker (1853)

Bittacus insularis E.-P. (1915)

Bittacus italicus (Müller) Klug (1838)

$=$ Panorpa italica Müller (1766)

$=$ Panorpa tipularia Fabricius (1775)

=Bittacus tipularius (Fabricius) Latreille (1805)

$=$ Panorpa tipuloides Schrank (1776)

Bittacus kagoshimaensis Issiki (1929b)

Bittacus kimminsi Tjeder (1956b)

Bittacus kunenensis Wood (1933)

Bittacus lachlani Navás (1925)

Bittacus laevipes Navás (1909)

Bittacus latipennis Gerstaecker (1885)

Bittacus leptocaudus Byers (1965a)

Bittacus leptocercus Navás (1934)

Bittacus lineatus Navás (1914)

=Bittacus similis Kimmins (1957)

Bittacus maculatus Issiki (1927)

Bittacus maculosus Byers (1965b)

Bittacus malaisei Tjeder (1974)

Bittacus marginatus Miyake (1913)

Bittacus mastrillii Navás (1913b)

Bittacus mexicanus Klug (1838)

Bittacus milleri Londt (1978)

Bittacus montanus Weele (1910)

=Bittacus woodi Smithers (1959)

Bittacus moschinus Navás (1914)

Bittacus nebulosus Klug (1838)

Bittacus nipponicus Navás (1909)

Bittacus nodosus Rust \& Byers (1976)

Bittacus occidentis Walker (1853)

=Bittacus arizonicus Banks (1911)

Bittacus omega Morgante (1967)

Bittacus oreinus Navás (1914)

Bittacus panamensis Byers (1958)

Taiwan

Kenya

Guyana

South Africa

China

Europe

Sri Lanka

Fr. Guinea

India

Sri Lanka

southern Europe

Japan

South Africa

Southwest Africa

Kenya

Japan

India

Thailand

Eastern Africa

Kenya

Taiwan

Trinidad

Burma

Japan

Japan

Mexico

South Africa

Angola, Cameroon, Kenya,

Malawi, Rhodesia,

Rwanda, Tanzania, Uganda

Malawi, Tanzania,

Zambia

Mozambique, South Africa

Japan

India, Pakistan

North America

Brazil

Ethiopia

Panama

Bittacus peringueyi E.P. (1913b)

South Africa 
Bittacus peterseni Kimmins (1938)

Bittacus pieii Navás (1935a)

Bittacus pignatelli Navás (1932a)

Bittacus pilicornis Westwood (1846)

Bittacus pinguipalpi Wood (1933)

Bittacus pintoi Souza Lopes \& Mangabeira (1942)

Bittacus planus Cheng (1949)

Bittacus pobequini (Navás) E.-P. (1913b)

$=$ Haplodictyus pobequini Navás (1908)

$=$ Bittacus kongoloensis Lestage (1929)

Bittacus pondoensis Londt (1972)

Bittacus punctiger Westwood (1846)

Bittacus rossi Londt (1977)

Bittacus schoutedeni E.P. (1913b)

$=$ Bittacus lestagei Kimmins (1957)

Bittacus selysi E.-P. (1917)

Bittacus sinensis Walker (1853)

=Diplostigma sinense (Walker) Navás (1908)

= Bittacus quaternipunctatus Enderlein (1910)

--Bittacus strategus Navás (1913b)

Bittacus sinicus Issiki (1931)

Bittacus sjostedti Weele (1910)

Bittacus smithersi Londt (1972)

Bittacus sobrinus Tjeder (1956b)

Bittacus solitarius Tjeder (1956b)

Bittacus sonani Issiki (1929b)

Bittacus stanleyi Byers (1968)

Bittacus stigmaterus Say (1823)

$=$ Bittacus pallidipennis Westwood (1846)

Bittacus striatus Issiki (1927)

Bittacus strigosus Hagen (1861) $=$ Leptobittacus strigosus (Hagen) Hine (1898)

Bittacus takaoensis Miyake (1913) =Haplodictyus polemicus Navás (1913c)

Bittacus taraiensis Penny (1969)

Bittacus testaceus Klug (1838) $=$ Bittacus brincki Tjeder (1956b)

Bittacus texanus Banks (1908)

Bittacus tienmushana Cheng (1957a)

Bittacus tjederi Londt (1970)

Bittacus triangularis Issiki (1929b)

Bittacus tuxeni Byers (1975b)

Bittacus vexilliferus Byers (1970)

Bittacus vumbanus Smithers (1960)

Bittacus wahlbergi Londt (1972)

Bittacus walkeri E.P. (1915)

$=$ Bittacus angulosus Tjeder (1956b)

Bittacus weelei E.-P. 1913b)

=Bittacus longistigma Navás (1926c)

=Bittacus negus Navás (1915)

$=$ Bittacus wittei Navás (1933b)
South Africa

China

Panama

eastern U.S.A.

Southwest Africa

Brazil

China

Congo, Ghana,

Ivory Coast, Nigeria

Uganda

South Africa

eastern N.A.

Zaïre

Zaïre

South Africa

eastern Asia

China

Kenya, Tanzania

South Africa

South Africa

Rhodesia

Taiwan

Zaïre

North America

Taiwan

North America

Japan

India

Rhodesia, South Africa

U.S.A.

China

South Africa

Korea, Manchuria

Guinea

China

Rhodesia

South Africa

South Africa

central Africa 
Bittacus zambezinus Navás (1931b) = Bittacus natalensis Wood (1933)

Bittacus zulu Londt (1972)

EDRIOBITTACUS Byers (1974)

Edriobittacus microcercus (Gerstaecker) Byers (1974) =Bittacus microcercus Gerstaecker (1885)

$=$ Kalobittacus microcercus (Gerstaecker) Riek (1954)

HARPOBITTACUS Gerstaecker (1885)

Harpobittacus australis (Klug) Gerstaecker (1885)

$=$ Bittacus australis Klug (1838)

= Bittacus corethrarius Rambur (1842)

$=$ Bittacus intermedius Selys (1868) (female)

Harpobittacus australis rubripes Riek (1954)

Harpobittacus brewerae Smithers (1973)

Harpobittacus limnaeus Smithers (1973)

Harpobittacus nigratus Navás (1932b)

Harpobittacus nigriceps (Selys) Gerstaecker (1885)

$=$ Bittacus nigriceps Selys (1868)

= Bittacus intermedius Selys (1868) (male)

=Harpobittacus intermedius (Selys) Gerstaecker (1885) (ma

Harpobittacus phaeoscius Riek (1954)

Harpobittacus rubricatus (Riek) Smithers (1973)

=Harpobittacus tillyardi rubricatus Riek (1954)

Harpobittacus scheibeli E.-P. (1935)

Harpobittacus similis E.-P. (1935)

Harpobittacus tillyardi E.-P. (1915)

Harpobittacus tillyardi albatus Riek (1954)

ISSIKIELLA Byers (1972)

Issikiella puichra Byers (1972)

KaLOBITTACUS E.P. (1914)

Kalobittacus bimaculatus E.P. (1914)

Kalobittacus hubbelli Byers (1965b)

Kalobittacus ramosus Byers (1958)

NANNOBITTACUS E.P. (1927)

Nannobittacus elegans E.-P. (1927)

Nannobittacus tjederi Byers (1965b)

NEOBITTACUS E.P. (1914)

Neobittacus aripuanaensis Penny (1977b)

Neobittacus blancheti (Pictet) E.-P. (1914)

=Bittacus blancheti Pictet (1836)

PAzIUS Navás (1913a)

Pazius flinti Byers (1977)

Pazius furcatus Byers (1957)

Pazius gracilis (Navás) Navás (1913a)

$=$ Bittacus gracilis Navás (1908)

Pazius obtusus Byers (1957)

Pazius ornaticaudus Penny (1977b)

Pazius pectinatus Byers (1977)
Mozambique, Malawi

Rhodesia, South Africa

South Africa

Australia

Australia, Tasmania

Australia

Australia

Australia

Australia

Australia

Australia

Australia

Australia

Australia

Australia

Australia

Brazil

Mexico

Honduras

Panama

Brazil

Colombia, Panama

Venezuela

Brazil

Brazil

Panama

Venezuela

Peru

Panama

Brazil

Ecuador 
TYтTновIтTACUS Smithers (1973)

Tytthobittacus macalpinei Smithers (1973)

Family BOREIDAE

BOREUS Latreille (1816)

$=$ Ateleptera Dalman (1823)
$=$ Euboreus Lestage (1940)

Boreus beybienkoi Tarbinsky (1962)

Boreus borealis Banks (1923)

=Euboreus borealis (Banks) Lestage (1940)

Boreus brumalis Fitch (1847)

=Euboreus brumalis (Fitch) Lestage (1940)

Boreus californicus Packard (1870)

$=$ Boreus unicoler Hine (1901)

=Euboreus unicolor (Hine) Lestage (1940)

$=$ Boreus isolatus Carpenter (1935)

=Euboreus isolatus (Carpenter) Lestage (1940)

=Boreus californicus fuscus Carpenter (1935)

=Euboreus californicus (Packard) Lestage (1940)

Boreus chagzhigireji Pliginsky (1914)

Boreus coloradensis Byers (1955)

Boreus elegans Carpenter (1935)

=Euboreus elegans (Carpenter) Lestage (1940)

Boreus hyemalis (Linnaeus) Latreille (1816)

$=$ Panorpa hyemalis Linnaeus (1767)

$=$ Gryllus proboscideus Panzer (1796)

$=$ Bittacus hiemalis (Linnaeus) Latreille (1805)

$=$ Boreus hiemalis (Linnaeus) Latreille (1817)

$=$ Ateleptera hiemalis (Linnaeus) Dalman (1823)

$=$ Boreus gigas Brauer (1876)

Boreus intermedius Lloyd (1934)

=Euboreus intermedius (Lloyd) Lestage (1940)

Boreus kratochvili Mayer (1938)

Boreus lokayi Klapálek (1901)

Boreus navasi Pliginsky (1914)

$=$ Boreus aktijari Pliginsky (1914)

Boreus nivoriundus Fitch (1847)

$=$ Euboreus nivoriundus (Fitch) Lestage (1940)

Boreus nix Carpenter (1935)

$=$ Euboreus nix (Carpenter) Lestage (1940)

=Boreus gracilis Carpenter (1935)

=Euboreus gracilis (Carpenter) Lestage (1940)

Boreus orientalis Martynova (1954)

Boreus pilosus Carpenter (1935)

=Euboreus pilosus (Carpenter) Lestage (1940)

Boreus reductus Carpenter (1933)

=Euboreus reductus (Carpenter) Lestage (1940)

Boreus semenovi Pliginsky (1930)

=Euboreus semenovi (Pliginsky) Lestage (1940)

Boreus sjostedti Navás (1926d)

Boreus vlasovi Martynova (1954)
U.S.S.R.

Alaska

eastern N.A.

western N.A.

\section{U.S.S.R.}

western N.A.

western N.A.

western Europe

Alaska

Czechoslavakia

Romania

U.S.S.R.

eastern N.A.

western N.A.

eastern U.S.S.R.

western N.A.

western N.A.

U.S.S.R.

U.S.S.R.

U.S.S.R. 
Boreus westwoodi Hagen (1866)

western Europe

=Boreus boldyrevi Navás (1911)

= Boreus tarnanii Navás (1911)

HESPEROBOREUS Penny (1977a)

Hesperoboreus brevicaudus (Byers) Penny (1977a)

western U.S.A. $=$ Boreus brevicaudus Byers (1961)

Hesperoboreus notoperates (Cooper) Penny (1977a)

western U.S.A.

= Boreus notoperates Cooper (1972)

Family CHORISTIDAE

CHORISTA Klug (1838)

$=$ Euphania Westwood (1846)

Chorista australis Klug (1838)

Chorista luteola (Westwood) Riek (1973)

=Euphania luteola Westwood (1846)

Australia

Australia

NEOCHORISTA Riek (1973)

Neochorista insolita Riek (1973)

Neochorista ruficeps (Newman) Riek (1973)

Australia

$=$ Panorpa ruficeps Newman (1850)

$=$ Chorista ruficeps (Newman) E.P. (1921)

$=$ Chorista victoriensis Issiki (1931)

TAENIOCHORISTA E.P. (1914)

Taeniochorista bifurcata Riek (1973)

Australia

Taeniochorista nigrita Riek (1973)

Australia

Taeniochorista pallida E.P. (1914)

Australia

Taeniochorista similis Riek (1973)

Australia

\section{Family MEROPEIDAE}

AUSTROMEROPE Killington (1933)

Austromerope poultoni Killington (1933)

Australia

MEROPE Newman (1838)

Merope tuber Newman (1838)

eastern U.S.A.

Family NANNOCHORISTIDAE

MICROCHORISTA Byers (1974)

$=$ Choristella Tillyard (1917) (preoccupied)

Microchorista philpotti (Tillyard) Byers (1974)

=Choristella philpotti Tillyard (1917)

New Zealand

NANNOCHORISTA Tillyard (1917)

Nannochorista dipteroides Tillyard (1917)

Nannochorista dipteroides eboraca (Tillyard) Riek (1954)

$=$ Nannochorista eboraca Tillyard (1917)

Nannochorista edwardsi Kimmins (1929)

Nannochorista holostigma Tillyard (1917)

Nannochorista maculipennis Tillyard (1917)

Australia, Tasmania

Australia

Argentina, Chile

Tasmania

Tasmania

Nannochorista neotropica Navás (1928a)

Chile 
NOTIOTHAUMA MacLachlan (1877)

Notiothauma reedi MacLachlan (1877)

Chile

Family PANORPIDAE

LEPTOPANORPA MacLachlan (1875)

$=$ Himanturella Enderlein (1910)

Leptopanorpa charpentieri (Burmeister) E.-P. (1915)

$=$ Panorpa charpentieri Burmeister (1839)

= Neopanorpa linguata Navás (1914]

= Leptopanorpa linguata (Navás) E.P. (1921)

Leptopanorpa cingulata (Enderlein) Byers (1967)

= Campodotecnum cingulatum Enderlein (1912)

= Neopanorpa cingulata (Enderlein) E.P. (1921)

=Leptopanorpa erythrura Lieftinck (1936)

Leptopanorpa filicauda Lieftinck (1936)

Leptopanorpa inconspicua Lieftinck (1936)

Leptopanorpa jacobsoni (Weele) E.-P. (1921)

$=$ Panorpa jacobsoni Weele (1909)

Leptopanorpa javanica (Westwood) E.P. (1921) Java

=Panorpa javanica Westwood (1842)

= Campodotecnum javanicum (Westwood) Enderlein (1910)

Leptopanorpa nematogaster (MacLachlan) MacLachlan (1875) Java

= Panorpa nematogaster MacLachlan (1869)

$=$ Neopanorpa nematogaster (MacLachlan) Weele (1909)

= Himanturella nematogaster (MacLachlan) Enderlein (1910)

=Leptopanorpa sieboldi MacLachlan (1875)

Leptopanorpa peterseni Lieftinck (1936)

Java, Sumatra

Java

Java

Java

Java

Leptopanorpa pi (Weele) Lieftinck (1936)

Java

=Panorpa pi Weele (1909)

$=$ Campodotecnum lemniscata Enderlein (1912)

= Neopanorpa lemniscata (Enderlein) E.P. (1921)

=Leptopanorpa brachyura Lieftinck (1934) (nomen nodum)

Lepiopanorpa pi decorata Lieftinck (1936)

Java

Leptopanorpa ritsemae MacLachlan (1875)

Java

=Leptopanorpa longicauda Weele (1909)

$=$ Himanturella tubifera Enderlein (1910)

Leptopanorpa robusta Lieftinck (1936)

Leptopanorpa sarangana Lieftinck (1936)

Java

Java

NEOPANORPA Weele (1909)

$=$ Campodotecnum Enderlein (1910)

Neopanorpa angustiapicula Chau \& Byers (1978)

Java

Neopanorpa angustipennis (Westwood) E.P. (1913c)

Burma

=Panorpa angustipennis Westwood (1842)

=Campodotecnum angustipennis (Westwood) Enderlein (1910)

Neopanorpa annamensis Byers (1965a)

Neopanorpa apicata Navás (1922)

Neopanorpa appendiculata (Westwood) E.-P. (1915) = Panorpa appendiculata Westwood (1846)

\section{Vietnam \\ China \\ India}

(*) Ponomarenko and Rasnitsyn (1974) have pointed out that since some fossil forms have recently been placed in this family, the family name should more properly be Eomeropidae. We prefer to retain the name Notiothaumidae pending further study of the fossil forms. 
Neopanorpa banksi Carpenter (1938)

Neopanorpa baviensis Cheng (1953)

Neopanorpa benaci Navás (1935b)

Neopanorpa borneensis Byers (1966)

Neopanorpa brisi (Navás) Carpenter (1938)

=Leptopanorpa brisi Navás (1930a)

=Neopanorpa pilosa Carpenter (1945)

Neopanorpa burmana Byers (1965a)

Neopanorpa cantonensis Cheng (1957a)

iveopanorpa carpenteri Cheng (1957a)

Neopanorpa cavaleriei (Navás) E.-P. (1921)

= Panorpa cavaleriei Navás (1908)

Neopanorpa caveata Cheng (1957a)

Neopanorpa chaoi Cheng (1957a)

Neopanorpa chelata Carpenter (1938)

Neopanorpa chillcotti Byers (1971a)

Neopanorpa choui Cheng (1949)

Neopanorpa claripennis Carpenter (1938)

Neopanorpa contracta Cheng (1953)

Neopanorpa cornuta E.-P. (1915)

Neopanorpa crinita Chau \& Byers (1978)

Neopanorpa cuspidata Byers (1965a)

Neopanorpa denticulata Rust \& Byers (1976)

Neopanorpa diloba Chau \& Byers (1978)

Neopanorpa dimidiata Navás [1930a)

Neopanorpa dispar Issiki \& Cheng (1947)

Neopanorpa dorsalis Byers (1965a)

Neopanorpa echinata Rust \& Byers (1976)

Neopanorpa effusa (Navás) Byers (1917a)

$=$ Campodotecnum effusum Navás (1913c)

=Leptopanorpa effusa (Navás) E.P. (1921)

Neopanorpa fenestrata (Needham) E.-P. (1915) $=$ Panorpa fenestrata Needham (1909)

Neopanorpa flava E.-P. (1915)

Neopanorpa flavicauda Banks (1930b)

Neopanorpa formosana (Navás) E.-P. (1915)

= Campodotecnum formosanum Navás (1911)

= Panorpa formosana (Navás) Miyake (1913)

Neopanorpa formosensis Navás (1930c)

Neopanorpa fractura Chau \& Byers (1978)

Neopanorpa furcata (Hardwicke) Byers (1971)

=Panorpa furcata Hardwicke (1825)

=Leptopanorpa furcata (Hardwicke) E.-P. (1915)

Neopanorpa fuscicauda Chau \& Byers (1978)

Neopanorpa gestroi Navás (1929)

Neopanorpa gibbosa Rust \& Byers (1976)

Neopanorpa gradana Cheng (1952)

Neopanorpa harmandi (Navás) Navás (1930c)

= Panorpa harmandi Navás (1908)

Neopanorpa harmandi conjuncta Navás (1930c)

Neopanorpa heii Cheng (1949)

Neopanorpa hirsuta (Crampton) Carpenter (1931b)

=Panorpa hirsuta Crampton (1931)
China

Vietnam

India

Borneo

India

Burma

China

China

China

China

China

China

Nepal

China

China

India

India

Sumatra

Thailand

India

Java

China

Taiwan

Vietnam

India

Nepal

India

Sikkim

Borneo

Taiwan

Taiwan

Sumatra

Nepal

Java

Burma

India

Taiwan

Thailand, Vietnam

Thailand

China

India 
Neopanorpa huangshana Cheng (1957a)

Neopanorpa hyaimata E.P. (1913)

= Campodiorecnum faicatum Navás (1913c)

Neopanorpa indica Rust \& Byers (1976)

Neopanorpa intuscata Banks (1930a)

Neopanorpa k-macuiata Cheng (1952)

Neopanorpa kwangtsehi Cheng (1957a)

Neopanorpa lacunaris Navás (1930a)

Neopanorpa latipennis Cheng (1949)

Neopanorpa lichuanensis Cheng (1957b)

Neopanorpa lieftincki Chau \& Byers (1978)

Neopanorpa lungtausana Cheng (1957b)

Neopanorpa maai Cheng (1957a)

Neopanorpa magna Issiki (1927)

Neopanorpa makii Issiki (1927)

Neopanorpa mokansana Cheng (1957b)

Neopanorpa mulleri (Weele) E.-P. (1915)

$=$ Panorpa mulleri Weele (1909)

=Panorpa mulleri ungaranensis Weele (1909)

Neopanorpa mutabilis Cheng (1957a)

Neopanorpa nielseni Byers (1965a)

Neopanorpa nigritis Carpenter (1938)

Neopanorpa nipalica (Navás) E.-P. (1915)

= Penorpa nipalica Navás (1910)

=Aulops suffusa Navás (1913b)

Neopanorpa ocellaris (Navás) E.P. (1921)

$=$ Panorpa ocellaris Navás (1908)

Neopanorpa ochrura Rust \& Byers (1976)

Neopanorpa ornata Byers (1965a)

Neopanorpa ovata Cheng (1957a)

Neopanorpa panda Byers (1965a)

Neopanorpa parva Carpenter (1945)

Neopanorpa parvula Willmann (1976)

Neopanorpa pendulifera Byers (1965a)

Neopanorpa pielina Navás (1936)

Neopanorpa puichra Carpenter (1945)

Neopanorpa ramulata Byers (1975a)

Neopanorpa salai Navás (1928b)

Neopanorpa sauteri (E.-P.) E.-P. (1915)

=Panorpa sauteri E.P. (1912)

Neopanorpa siamensis Byers (1965a)

Neopanorpa sordida (Needham) E.P. (1915)

$=$ Panorpa sordida Needham (1909)

Neopanorpa spatulata Byers (1965a)

Neopanorpa spicata Byers (1966)

Neopanorpa sumatrana Chau \& Byers (1978)

Neopanorpa taoi Cheng (1949)

Neopanorpa thai Byers (1965a)

Neopanorpa tienmushana Cheng (1957a)

- Neopanorpa tiomanensis Byers (1966)

Neopanorpa translucida Cheng (1957a)

Neopanorpa tuberosa Byers (1965a)
China

Java

India

Malaysia, Thailand

Taiwan

China

China

China

China

Sumatra

China

China

Taiwan

Taiwan

China

Java

China

Vietnam

China

India, Sikkim

Sìkkim

India

Vietnam

China

Vietnam

China

Vietnam

Thailand

China

China

Nepal

India

Taiwan

Thailand

India

Thailand

Borneo

Sumatra

China

Thailand

China

Tioman Is.

China

Thailand 
Neopanorpa umbonata Chau \& Byers (1978)

Sumatra

Neopanorpa validipennis Cheng (1949)

China

Neopanorpa varia Cheng (1949)

China

Neopanorpa vietnamensis Willmann (1976)

Vietnam

Neopanorpa zebrata E.-P. (1915)

India

JANORPA Linnaeus (1758)

= Panorpha (sic) Radermacher (1779)

$=$ Aulops Enderiein (1910)

=Estenaila Navás (1912)

Panorpa acuta Carpenter (1931a)

U.S.A.

Panorpa akasakai Issiki (1929b)

Taiwan

Panorpa alpina Rambur (1842)

=Aulops alpina (Rambur) Enderlein (1910)

=Panorpa variabilis Brauer (1857)

Panorpa americana Swederus (1787)

= Panorpa fasciata Fabricius (1793)

Panorpa amurensis MacLachlan (1872)

=Aulops amurensis (MacLachlan) Enderlein (1910)

Panorpa angustistriata Issiki (1929b)

Panorpa annexa MacLachlan (1869)

=Panorpa ghilianii MacLachlan (1869)

= Panorpa adnexa (sic) Navás (1927)

Panorpa annexa etrusca Willmann (1976c)

Panorpa anomala Carpenter (1931a)

= Panorpa proximata Carpenter (1931a)

Panorpa antiporum Nagler (1968)

Panorpa apiconebulosa Issiki (1929b)

Panorpa approximata E.P. (1915)

Panorpa arakavae Miyake (1913)

Panorpa arcuata (Navás) E.-P. (1915)

=Estenalla arcuata Navás (1912)

Panorpa aspoecki Willmann (1973)

Panorpa aurea Cheng (1957a)

Panorpa azteca Byers (1958)

Panorpa banksi Hine (1901)

= Panorpa affinis Banks (1895) (preoccupied)

$=$ Panorpa chelata Carpenter (1931a)

Panorpa banksiana Penny \& Byers $n$. novum =Panorpa interrupta Banks (1918) (preoccupied)

Panorpa baohwashana Cheng (1957a)

Panorpa bicornuta MacLachlan (1887) =Aulops bicornuta (MacLachlan) Enderlein (1910) = Panorpa magnicauda Miyake (1910)

Panorpa bifida Carpenter (1935)

Panorpa bistriata Issiki (1929b)

Europe

U.S.A.

eastern Asia

Taiwan

France, Germany

Italy

U.S.A.

Romania

Taiwan

Korea

Japan

U.S.S.R.

Turkey

China

Mexico

U.S.A.

U.S.A.

China

Japan

Panorpa bonis Cheng (1949)

Panorpa braueri Carpenter (1931a)

Panorpa brevititilana Issiki (1929b)

Panorpa bunun Issiki (1929b)

Panorpa carolinensis Banks (1905)

U.S.A.

Taiwan

China

U.S.A.

Taiwan

Taiwan

U.S.A.

China

Panorpa carpenteri Cheng (1957a)

Iran

=Aulops caucasica (NacLachlan) Enderlein (1910) 
Panorpa centralis Tjeder (1936)

China

Panorpa cheni Cheng (1957a)

China

Panorpa chiensis Cheng (1953)

Korea

Panorpa cladocerca Navás (1935a)

China

Panorpa claripennis Hine (1901)

U.S.A.

Panorpa clavigera Klapálek (1902)

Asia, Europe

Panorpa cognata Rambur (1842)

Panorpa cognata osellai Willmann (1976c)

Panorpa communis Linnaeus (1758)

= Pancrpa vulgaris Imhoff \& Labram (1845)

Panorpa communis raehlei Lauterbach (1970)

Panorpa concolor E.P. (1915)

Panorpa connexa MacLachlan (1869)

Panorpa consuetudinis Snodgrass (1927)

=Panorpa elaborata Carpenter (1931a)

Panorpa coomani Cheng (1957a)

Panorpa coreana Okamoto (1925)

Panorpa cornigera MacLachlan (1887) $=$ Auiops cornigera (MacLachlan) Enderlein (1910)

=Panorpa galloisi Miyake (1911) (homonym) new synonomy

= Panorpa galloisi Navás (1913) (homonym)

Panorpa cornigera fulvicaudaria Miyake (1910)

Panorpa curva Carpenter (1938)

Panorpa davidi Navás (1908)

Panorpa debilis Westwood (1846) = Panorpa canadensis Banks (1895)

Panorpa deceptor E.-P. (1913a)

Panorpa diceras MacLachlan (1894) =Panorpa grahami Carpenter (1938)

Panorpa difficilis Carpenter (1938) western Europe

Italy

Asia, Europe

Europe

Taiwan

Caucasus Mts.

U.S.A.

China

Korea

Japan

Panorpa dissimilis Carpenter (1931a)

Japan

China

China

U.S.A.

Panorpa dubitans Carpenter (1931a)

Panorpa emarginata Cheng (1949)

Panorpa esakii Issiki (1929b)

Panorpa falsa Issiki \& Cheng (1947)

Panorpa flavicorporis Cheng (1957a)

Taiwan

China

Panorpa fiavipennis Carpenter (1938)

China

U.S.A.

U.S.A.

China

Taiwan

Taiwan

China

China

Panorpa flexa Carpenter (1935)

U.S.A.

Panorpa fructa Cheng (1949)

Panorpa fukiensis Tjeder (1951)

China

China

Panorpa galerita Byers (1962a)

North America

Panorpa germanica Linnaeus (1758)

=Panorpa affinis Leach (1815)

= Panorpa apicalis Stephens (1835)

= Panorpa montana Brauer (1857)

= Panorpa gibberosa MacLachlan (1869)

$=$ Panorpa ruizi Navás (1926e)

=Panorpa pirioni Navás (1929) (nomen nudum)

Panorpa germanica riegeri Lauterbach (1971)

Panorpa germanica euboica Lauterbach (1972)

Panorpa germanica graeca Lauterbach (1972)

Panorpa germanica rumelica Lauterbach (1972)

western Europe

Germany

Balkans of

southern Europe

Greece

Turkey 
Panorpa gokaensis Miyake (1910)

Panorpa gracilis Carpenter (1931a)

Panorpa grahamana Cheng (1957a)

Panorpa gressitti Byers (1970)

Panorpa guttata Navás (1908)

Panorpa hageniana (Willmann) Willmann (1978)

$=$ Panorpa turcica hageniana Willmann (1975)

Panorpa hakusanensis Miyake (1913)

Panorpa hamata 'ssiki \& Cheng (1947)

Panorpa helena Byers (1962a)

= Panorpa venosa authors, not Westwood (1846)

Panorpa horiensis Issiki (1929a)

Panorpa horni Navás (1928)

Panorpa hungerfordi Byers (1973)

Panorpa hybrida MacLachlan (1882)

$=$ Panorpa romanica Bechet $(1955)$

Panorpa immaculata E.-P. (1915)

Panorpa implicata Cheng (1957a)

Panorpa indivisa Martynova (1957)

Panorpa insolens Carpenter (1935)

Panorpa isolaťa Carpenter (1931a)

$=$ Panorpa decorata Carpenter (1931a)

Panorpa issikiana Byers (1970)

Panorpa issikii Penny \& Byers $n$. novum $=$ Panorpa acuta Issiki \& Cheng (1947) (preoccupied)

Panorpa japonica Thunberg (1784)

$=$ Aulops japonica (Thunberg) Enderlein (1910)

= Panorpa macrogaster MacLachlan (1867)

=Aulops macrogaster (MacLachlan) Enderlein (1910)

= Panorpa japonica macrogaster (MacLachlan) Miyake (1913)

$=$ Panorpa irreguiaris Miyake (1910)

= Panorpa niphonensis Miyake (1908)

= Panorpa pulchra Miyake (1908)

=Aulops pulchra (Miyake) Enderlein (1910)

= Panorpa rectifasciata Miyake (1908)

=Aulops rectifasciata (Miyake) Enderlein (1910)

$=$ Panorpa sinanoensis Miyake (1908)

=Aulops sinanoensis (Miyake) Enderlein (1910)

= Panorpa dyscola Navás (1908) (female)

= Panorpa hageni Navás (1909)

=Aulops interrupta Navás (1913)

= Penorpa leucothyria Navás (1908)

=Aulops leucothyria (Navás) Navás (1911)

Panorpa kamikotiensis Issiki (1929a)

Panorpa kellogi Cheng (1957b)

Panorpa kimminsi Carpenter (1948)

Panorpa kirismaensis Issiki (1929a)

Panorpa kiusiuensis Issiki (1929b)

Panorpa klapperichi Tjeder (1951)

Panorpa klugi MacLachlan (1867)

=Aulops klugi (MacLachlan) Enderlein (1910)

= Panorpa ochracea Miyake (1908)

=Aulops ochracea (Miyake) Enderlein (1910)

= Panorpa drouarti Navás (1908)

=Aulops drouarti (Navás) Navás (1911)
Japan

U.S.A.

China

China

Tibet

Turkey

Japan

Taiwan

U.S.A.

Taiwan

U.S.S.R.

U.S.A.

eastern Europe

Mexico

China

eastern U.S.S.R.

U.S.A.

U.S.A.

China

Taiwan

Japan
Japan

China

China

Japan

Japan

China

Japan 
= Panorpa dyscola Nayás (1908) (male)

=Aulops dyscola (Navás) Navás (1911)

$=$ Aulops trisignata Navás (1913b)

Panorpa komaensis Okamoto (1925)

Korea

Panorpa kongosana Okamoto (1925)

Korea

Panorpa lacedaemonia Lauterbach (1972)

Greece

$=$ Panorpa germanica corcyraea (Lauterbach) Ward (1978)

Taiwan

Panorpa lachlani Navás (1930d)

U.S.A.

$=$ Panorpa longipennis Banks (1911)

Panorpa leei Cheng (1949)

China

Panorpa leucoptera Uhler (1858)

Japan

=Aulops leucoptera (Uhler) Enderlein (1910)

$=$ Panorpa nikkoensis Miyake (1908)

=Aulops nikkoensis (Miyake) Enderlein (1910)

Panorpa lewisi MacLachian (1887)

=Aulops lewisi (MacLachlan) Enderlein (1910)

$=$ Panorpa chuzenjiensis Miyake (1910)

Panorpa lintienshana Cheng (1952)

Taiwan

Panorpa longicornis Carpenter (1931a)

Panorpa longiramina Issiki \& Cheng (1947)

Panorpa longititilana Issiki (1929b)

U.S.A.

Taiwan

Taiwan

Panorpa lugubris Swederus (1787)

U.S.A.

$=$ Aulops lugubris (Swederus) Enderlein (1910)

$=$ Panorpa scorpio Fabricius (1793)

=Bittacus scorpio (Fabricius) Latreille (1807)

Panorpa lutea Carpenter (1945)

China

Panorma maculosa Hagen (1851)

Panorpa meridionalis Rambur (1842)

Panorpa mexicana Banks (1913)

Panorpa mirabilis Carpenter (1931a)

Panorpa mokansana Cheng (1957b)

Panorpa multifasciaria Miyake (1910)

Panorpa navasi Issiki (1929a)

Panorpa nebulosa Westwood (1846)

Panorpa neglecta Carpenter (1931a)

Panorpa nigrirostris MacLachlan (1882)

=Panorpa selysi E.P. (1915)

Panorpa nipponensis Navás (1908)

=Aulops nipponensis (Navás) Navás (1911)

= Panorpa brachypennis Miyake (1908)

=Aulops brachypennis (Miyake) Enderlein (1910)

=Aulops dentata Navás (1913)

=Aulops picea Navás (1913b)

$=$ Aulops valignanii Navás (1913b)

=Panorpa klugi maculata Miyake (1913)

=Panorpa klugi quadrimaculata Miyake (1913)

Panorpa nokoensis Issiki (1929b)

Taiwan

Panorpa nuptialis Gerstaecker (1863)

U.S.A.

Panorpa obliqua Carpenter (1945)

China

Panorpa obscura Miyake (1910)

Japan

China

Panorpa obtusa Cheng (1949)

Taiwan

Panorpa ochraceocauda Issiki (1927)

Japan 
Panorpa okamotoana Issiki (1927)

Panorpa ophtalmica (Navás) Miyake (1913)

= Neopanorpa ophthalmica Navás (1911)

Panorpa orientalis MacLachlan (1887)

=Aulops melania Navás (1912)

= Panorpa sachalinensis Matsumura (1911)

Panorpa pallidimaculata Issiki (1929b)

Panorpa palustris Byers (1958)

Panorpa pectinata Issiki (1929b)

Panorpa penicillata Byers ( $1962 b)$

Panorpa peterseana Issiki (1927)

Panorpa picta Hagen (1863)

Panorpa pieli Cheng (1957a)

Panorpa pieperi Willmann (1975)

Panorpa plitvicensis Lauterbach (1972)

Panorpa pryeri MacLachlan (1875)

=Aulops preyeri (MacLachlan) Enderlein (1910)

= Panorpa bouvieri Navás (1908)

=Aulops bouvieri (Navás) Navás (1911)

Panorpa preyeri major Miyake (1908)

Panorpa pseudoalpina Nagler (1970)

Panorpa punctata Klug (1838)

Panorpa pura Klapáiek (1906)

Panorpa pusilla Cheng (1949)

Panorpa rantaisanensis Issiki (1929b)

Panorpa robusta Carpenter (1931a)

Panorpa rufa Gray (1832)

Panorpa rufescens Rambur (1842)

=Panorpa venosa Westwood (1846)

=Panorpa confusa Westwood (1846)

Panorpa rufostigma Westwood (1846)

Panorpa schweigeri Willmann (1975)

Panorpa semifasciata Cheng (1949)

Panorpa setifera Webb (1974)

Panorpa sexspinosa Cheng (1949)

Panorpa shibatai Issiki (1929b)

Panorpa similis E.P. (1915)

Panorpa sigmoides Carpenter (1931a)

Panorpa similis E.P. (1015)

Panorpa sonani Issiki (1929b)

Panorpa speciosa Carpenter (1931a)

Panorpa statura Cheng (1949)

Panorpa stigmalis Navás (1908)

= Campodotecnum stigmalis (Navás) Navás (1911)

Panorpa stotzneri E.P. (1934)

Panorpa striata Miyake (1908) =Aulops striata (Miyake) Enderlein (1910)

Panorpa subfurcata Westwood (1846)

$=$ Panorpa signifer Banks (1900)

= Panorpa modesta Carpenter (1931a)

Panorpa submaculosa Carpenter (1931a)

= Panorpa utahensis Gurney (1937)

Panorpa subulifera Byers (1962b)
Korea

Taiwan

Japan

Taiwan

eastern U.S.A.

Taiwan

Mexico

Taiwan

Causasus

China

Turkey

Yugoslavia

Japan

Japan

Romania

Mexico

Asia, Europe

China

Taiwan

U.S.A.

U.S.A.

U.S.A.

Albania

Turkey

China

U.S.A.

China

Taiwan

U.S.S.R.

U.S.A.

Caucasus

Taiwan

U.S.A.

China

Tibet

China

Japan

U.S.A.

U.S.A.

U.S.A. 


\begin{tabular}{ll} 
Panorpa susteri Nagler (1970) & Romania \\
Panorpa taiheisanensis Issiki (1929a) & Taiwan \\
Panorpa taiwanensis Issiki (1929b) & Taiwan \\
Panorpa takenouchii Miyake (1908) & Japan \\
$\quad=$ Aulops takenouchii (Miyake) Enderlein (1910) & \\
Panorpa tatvana Willmann (1974) & Turkey \\
Panorpa tatvana ressli Willmann (1975) & Turkey \\
Panorpa terminata Klug (1838) & Mexico \\
Panorpa tetrazonia Navás (1935a) & China \\
Panorpa thompsoni Cheng (1957b) & China \\
Panorpa thrakica Willmann (1976) & European Turkey \\
Panorpa tincta Navás (1931a) & China \\
Panorpa titschacki E.-P. (1934) & Greece \\
Panorpa tjederi Carpenter (1938) & China \\
Panorpa trifasciata Cheng (1957a) & China \\
Panorpa trizonata Miyake (1908) & Japan \\
=Aulops trizonata (Miyake) Enderlein (1910) & \\
Panorpa tsunekatanus Issiki (1929b) & Japan \\
Panorpa turcica Willmann (1975) & Turkey \\
Panorpa turcica anatolica Willmann (1975) & Turkey \\
Panorpa turcica pontica Wi!lmann (1975) & Turkey \\
Panorpa typicoides Cheng (1949) & China \\
Panorpa vernalis Byers (1973) & U.S.A. \\
Panorpa virginica Banks (1906) & U.S.A. \\
Panorpa waongkehzengi Navás (1935a) & China \\
Panorpa wormaldi MacLachlan (1875) & Japan \\
=Aulops wormaldi (MacLachlan) Enderlein (1910) & \\
Panorpa wrighti Cheng (1957b) & China \\
Panorpa yiei Issiki \& Cheng (1947) & Taiwan \\
\hline
\end{tabular}

\section{Family PANORPODIDAE}

BRACHYPANORPA Carpenter (1931a)

Brachypanorpa carolinensis (Banks) Carpenter (1931a) U.S.A. $=$ Panorpodes carolinensis Banks (1905)

Brachypanorpa jaffersoni Byers (1976)

U.S.A.

Brachypanorpa montana Carpenter (1931a) U.S.A.

Brachypanorpa oregonensis (MacLachlan) Carpenter (1931a) U.S.A. =Panorpodes oregonensis MacLachlan (1881)

PANORPODES MacLachlan (1875)

Panorpodes apicalis Miyake (1910) Japan =Panorpa naevia Navás (1909)

Panorpodes decorata MacLachlan (1887) Japan $=$ Panorpodes notata Navás (1909)

Panorpodes decorata confusa Miyake (1913) Japan

Panorpodes decorata limbata (Navás) Miyake (1913) $=$ Panorpa limbata Navás (1909)

Panorpodes decorata singularis (Miyake) Miyake (1913) Japan $=$ Panorpodes singularis Miyake (1910)

Panorpodes komaensis Okamoto (1925) Korea

Panorpodes paradoxa MacLachlan (1875) Japan

Panorpodes paradoxa stigmatica Miyake (1913) Japan

Panorpodes pulchra Issiki (1927) Japan 


\section{Resumo}

Citamos de todas as espécies conhecidas de Mecoptera, com as citaçŏes bibliográficas originais e respectiva distribuição geográfica. Dados sobre a diversidade das famílias, gêneros e espécies são apresentados. bem como notadas duas homonimias e uma sinonímia.

\section{LITERATURE CITED (")}

Banks, NathaN

1895 - New Neuropteroid insects. Trans. Am. ent. Soc. 22:313-316.

1900 - New genera and species of Nearctic neuropteroid insects. Trans. Am. ent. Soc. 26:239-259.

1905 - Descriptions of new species of neuropterous insects from the Black Mountains, N.C. Bull. Am. Mus, nat. Hist. 21:215-218.

1906 - Three new species of Neuroptera. Psyche, Camb. 13:98-100

1908 - Neuropteroid insects. Notes and descriptions. Trans. Am. ent. Soc. 34:255-267.

1911 - Descriptions of new species of North American neuropteroid insects. Trans. Am. ent. Soc. $37: 335-360$.

1913 - Synopsis and descriptions of exotic Neuroptera. Trans. Am. ent. Soc. 39:201-242.

1918 - New neuropteroid insects. Bull. Mus. comp. Zool. Harv. 62:1-22.

1923 - Mecoptera. In McAtee, Insects, Arachnida, and Chilopods of the Probilof Islands, Alaska. U.S.D.A., Bureau of Biological Survey, N. Am. Fauna. 46:158.

1930 a - Some neuropteroid insects from the Malay peninsula. J. fed. Malay St. Mus. $16: 377-409$

$1930 \mathrm{~b}$ - Some neuropteroid insects from North Borneo, particularly from Mt. Kinabalu, 13.455 ft. J. fed. Malay St. Mus. 16:411-429.

BECHET, I.

1955 - Specii de Panorpate in Fauna R.P.R. (Nota I). Studii si cercetari stiintifice. Filiala Cluj, Academia R.R.R., Ser. 2 Stiinte biologica agricole si medicale. 6:53-57.

BRAUER, F.

1860 - Bittacus hageni, ein neue europaische Art, beschrieben und mit den verwandten Arten verglichen. Verh. zool. bot. Ges. Wien. 10:691-696
BraUer, $\mathrm{F}$.

1876 - Die Neuropteren Europas und insbesondere Oesterreichs mit Ruchsicht auf ihre geographische Verbreitung. Festschr. zur Feier d. 25. jahr. Bestehens d. k.k. zool.bot. Ges. 1876:263-300.

Brauer, F. \& Löw, F.

1857 - Neuroptera austriaca. 1-80. Wien

BURMEISTER, $\mathrm{H}$

1839 - Handbuch der Entomologie. Bd. 2, Teil 2. 757-1050. Berlin.

BYERS, G.W.

1955 - A new species of Boreus (Mecoptera : Boreidae) from Colorado. Occ. Pap. Mus. Zool. Univ. Mich. 562:1-4.

1957 - The neotropical genus Pazius (Mecoptera Bittacidae). Occ. Pap. Mus. Zool. Univ. Mich. 582:1-7

1958 - Descriptions and distributional records of American Miecoptera. J. Kans. ent. Soc. 31:213-222.

1961 - An unusual new species of Boreus (Mecoptera : Boreidae) from Oregon. J. Kans. ent. Soc. 34:73-78.

1962a - Type specimens of nearctic Mecoptera in European museums, including descriptions of new species. Ann. ent. Soc. Am 55:466-476.

1962b- Descriptions and distributional records of American Mecoptera. II. J. Kans. ent. Soc. 35:299-307.

1965a - The Mecoptera of Indo-China. Pacif. Insects. $7: 705-748$.

$1965 \mathrm{~b}$ - New and uncommon Neotropical Mecoptera. J. Kans. ent. Soc. 38:135-144.

1965c-Families and genera of Mecoptera. Proc. XII. int. Congr. Ent., London 1964. P. 123.

1966 - Mecoptera from Borneo and Tioman Island. Pacif. Insects. 8:885-892.

1968 - A new Bittacus from the Congo (Mecoptera: Bittacidae). J. Kans. ent. Soc. 41:34-36.

1970 - New and little known Chinese Mecoptera. J. Kans. ent. Soc. 43:383-394 .

1971a- A new Neopanorpa from Nepal. J. Kans. ent. Soc. 44:534-539.

$1971 \mathrm{~b}$ - An illustrated, annotated catalogue of African Mecoptera. Kans. Univ. Sci. Bull. 49:389-436.

(*) Abbreviations of journal names are in accordance with World List of Scientific Periodicals, whenever possible. Publication dates for articles are in accordance with Schlee \& Schlee (1976). 
1972 - A new genus of Mecoptera from Brazil. J. Kans. ent. Soc. 45:340-346.

1973 - Descriptions and distributional records of American Mecoptera. III. J. Kans. ent. Soc. 46:362-375

1974 - New generic names for Mecoptera of Australia and New Zealand. J. Austr. ent. Soc. 13:165-167.

1975a- Ergebnisse der Bhutan Expedition 1972 des Naturhistorischen Museums in Basel. Mecoptera: Fam. Panorpidae. Entomologica Basiliensia. 1:87-91.

1975b- Descriptions and distributional records of African Mecoptera, J. ent. Soc. sth Afr. 38:217-222.

1976 - A new Appalachian Brachypanorpa (Mecoptera: Panorpodidae). J. Kans, ent. Soc. 49:433-440

1977 - Revision of the genus Pazius (Mecoptera : Bittacidae). Revta. Biol. tropical. 25:109-121.

CAPRA, F.

1939 - Planipennia, Mecoptera. Missione biol. Paese dei Borana. 13:157-178.

CARPENTER, F.M.

1931a- Revision of the Nearctic Mecoptera. Buli. Mus. comp. Zool. Harv. 72:205-277.

1931b- Neopanorpa hirsuta (Crampton). Psyche, Camb. 38:184-185.

1933 - A new Boreus from British Columbia (Me. coptera). Can Ent. 65:94-95.

1935 - New Nearctic Mecoptera, with notes on other species. Psyche, Camb. 42:105-122.

1938 - Mecoptera from China, with descriptions of new species. Proc. ent. Soc. Wash. 40:267-281.

1941 - A new genus of Mecoptera from Tasmania. Pap. Proc. R. Soc. Tasm. 1940:51-53.

1945 - Panorpidae from China (Mecoptera). Psyche, Camb. 52:70-78.

1948 - Notes on Chinese Panorpidae (Mecoptera). Psyche, Camb. 55:28-30.

Chau, H. C.S. \& Byers, G.W.

1978 - The Mecoptera of Indonesia: genus Neopanorpa. Univ. Kans. Sci. Bull. 51:341-405.

Cheng, F.Y.

1949 - New species of Mecoptera from Northwest China. Psyche, Camb. 56:139-173.

1952 - Additions to the Mecopterous fauna of For- mosa. Psyche, Camb. 59:89-95.

1953 - Three new species of Panorpidae (Mecoptera). Psyche, Camb. 60:119-122. 1957a- Revision of the Chinese Mecoptera. Bull. Mus. comp. Zool. Harv. 116:1-118.

1957b- Descriptions of new Panorpidae (Mecoptera) in the collection of the California Academy of Sciences. Mem. Coll. Agris natn. Taiwan Univ. 5:27-33.

COOPER, K.W.

1972 - A southern California Boreus, B. notope. rates n. sp. I. Comparative morphology and systematics. (Mecoptera: Boreidae). Psyche, Camb. 79:269-283.

DALMAN, J.W.

1823 - Analecta entomologica. PP. 1-104. Holmiae.

ENDERLEIN, G

1910 - Uber die Phylogenie und Klassifikation der Mecopteren unter Berucksichtigung der fossilen Formen. Zool. Ang. 35:385-399.

1912 - Zur Kenntnis der Mecopteren Javas. Notes Leyden Mus. 34:235-239.

ERICHSON, W.F.

1848 - Insecten. Pp. 553-617 In Schomburgk, R. Reisen in Brituschthürama in den Jahren 1840-1844. Bd. 3, Versuch einer Fauna und Flora von Britisch-Guiana. 533-1260. Liepzig.

EsBen-Petersen, P.

1912 - H Sauters Formosa-Ausbeute: Neuroptera Planipennia. Ent. Mitt. 1:197-198.

1913a- H. Sauter's Formosa-Ausbeute. Planipennia II, Megaloptera and Mecoptera. Ent. Mitt. 2:222-228, 257-265.

1913b- Notes on African Bittacidae. Revue zool. afr. 3:135-145

1913c - Mecoptera and Planipennia collected in Java by Edward Jacobson. Notes Leyden Mus. 35:225-236.

1914 - New genera and species of Mecoptera. Ent. Meddr. 10:129-132.

1915 - A synonymic list of the order Mecoptera together with descriptions of new species. Ent. Meddr. 10:216-242.

1917 - Two species of Bittacidae (Neuroptera) from South Africa. Ann. S Afr. Mus. 17:187-190.

1921 - Mecoptera. Monographic Revision Coll. zool. E. de Selys Longchamps. 5:1-172.

1927 - New and little-known species of Mecopte$\mathrm{ra}$ and Neuroptera in the Zoological Museum of Helsingfors. Notul. ent. 7:13-18.

1934 - Two new species of Panorpa Linn (Mecoptera). Vidensk. Meddr. dansk naturh. Foren. 97:2113-213 
1935 - Two new species of Harpobittacus Gerst. (Mecoptera). Vidensk. Meddr. dansk na. turh. Foren. 99:1-3.

FABRICIUS, J.C.

1775 - Systema Entomologiae. Pp. 1-832. Flens. burgi, Lipsiae.

1793 - Entomologia systematica emendata et aucta. Bd. 2. Pp. 1-519. Hafniae, Proft.

FrTCH, A.

1847 - Winter insects of eastern New York. Am J. Agric. Sci. 5:274-284.

Gerstaecker, A.

1863 - Uber einige neue Planipennien aus den Familien Hemerobiiden und Panorpiden. Stet. tin. ent. Ztg. 24:168-188.

1885 - Uber einige Arten der Gattung Bittacus Latr. Mitt. naturw. Ver. Neu-Vorpomm. 16:117-121.

Gray, G.R.

1832 - New species of insects of all the orders. In Griffith, E. The Animal kingdom arranged in conformity with its organization by the Baron Cuvier. Bd. 14:1-570; Bd. 15:1-769.

GURNEY; A.B.

1937 - A new species of Panorpa from Utah, with notes on other Nearctic species (Mecoptera). Proc, ent. Soc, Wash 39:222-227.

Hagen, H.A.

1861 - Synopsis of the Neuroptera of Nourth America with a list of the South American species. Smithson. misc. Collns. 4:1-347.

HARDWICKE, $T$

1825 - Description of the Cermatia longicornis and of three new insects from Nepaul. Trans. Linn. Soc. Lond. 14:131-136.

HINE, J.S.

1898 - The North American species of the genus Bittacus. J. Columbus hort. Soc. 13:105-115.

1901 - A review of the Panorpidae of America north of Mexico. Bull. scient. Labs Denison Univ. 11:241-264.

IssIKI, $\mathrm{S}$

1927 - New and rare species of Mecoptera from Corea, Formosa and Japan. Insecta mat. sum. 2:1-12.

1929 - Descriptions of new species of the genus Panorpa from Japan and Formosa. J. Soc. trop. Afric. 1:182-191.

$1929 \mathrm{~b}$ - Descriptions of new Mecoptera from the Japanese Empire. Trans. nat. Hist. Soc. Taiwan. 19:260-314.

1931 - Two new species of scorpion flies (Insecta, order Mecoptera). Ann. Mag. nat. Hist, $7: 219-222$.
Issiki, S. \& CHENG, F.Y.

1947 - Formosan Mecoptera with descriptions of new species. Mem. Coll. Agric. natn. Taiwan Univ. 1:1-17

Kaltenbach, A.

1978 - Mecoptera. Handbuch der Zoologie 4(2) 2/28:1-111

KILLINGTON, F.J

1933 - A new genus and species of Meropeidae (Mecoptera) from Australia. Entomologist's mon. Mag. 69:1-4.

KIMMINS, D.E.

1928 - Two new Bittacidae (Mecoptera). Ann. Mag. nat. Hist. (Ser. 10), 1:395-396.

1929 - Some new and little known Argentine Neuroptera. Revta Soc. ent. argent. 2:187-192.

1938 - Some new African Neuroptera and Mecoptera. Ann. Mag. nat. Hist. (Ser. 11). 1:289-294.

1957 - New species of East African Neuroptera. Mecoptera and Trichoptera. Ann. Mag. nat. Hist. (Ser, 12), 10:36-41.

KLAPÁLEK, F.

1901 - A novych a malo znamych druzich paiaearktickych Neuropteroid. Bulletin international (Sciences, mathematiques, et naturelles) Ceská akademie cisare Frantiska Josefa pro védy, slovesnost a umeni v Praze. 10:1-19.

1902 - Zur Kenntniss der Neuropteroiden von Ungarn, Bosnien und Herzegovina Természetr. Füz. 25:161-180.

1906 - Prispevek ke znalosti fauny Neuropteroid Chorvatska, Slavonska i zemi sousednich. Ceska akademie cisare Frantiska Josefa pro védy, slovesnost a umeni $v$ Praze. 15:1-8.

KLUG, F.

1838 - Versuch einer systematischen Feststellung der Insecten-Familie: Panorpatae und Auseinandersetzung ihrer Gattungen und Arten. Abh. preuss. Akad. Wiss. (1836). 1838:81-108

Latreille, P.A.

1805 - Histoire naturelle, générale et particulière. des Crustacés et des Insectes. Bd, 13:1-432.

1807 - Genera crustaceorum et insectorum secondum ordinem naturalem in familias dispo. sita, iconibus exemplisque plurimis expli. cata. Bd 2, 1-280. Parisii et Argentorat.

1816 - L'histoire générale et particulière des crustacés, des arachnides et des insectes. In Latreille, P.A. et. al. Nouveau dictionnaire d' histoire naturelle. Bd. 4:1-602.

1817 - Insectes. In Cuvier, G. Ch. L.D. Le Rè. gne Animale. Bd. 3. 
LAUTERBACH, K.E.

1970 - Eine neue Rasse von Panorpa communis L. aus Piemont, zugleich ein Beitrag zur Kennthis von Panorpa vulgaris Imhoff und Labram (Ins. - Mec.). Jh. Ges. Naturk. Wurttemberg. 125:284-290.

1971 - Eine neue Unterart von Panorpa germanica L. aus Bosnien (Mecoptera, Panorpidae). Ent. Z. Frankf. a. M. 81:65-68.

1972 - Zur Kenntnis der Skorpionsfliegen des Bał kans (Mecoptera, Panorpidae). Dt. ent. $\mathbf{Z}$. 19:109-140.

Lestage, J.A.

1929 - Notes critiques et zoogéographiques sur les Bittacus africains (Mecoptera). Revue Zool. Bot. afr. 18:1-22.

1940 - Pour I'histoire des Boreus (StégoptèresMécoptères). I. Partie. Annuls. Soc. r. zool. Belg. 71:271-320.

LIEFTINCK, M.A.

1934 - Uit het leven der scorpioenvliegen (Panorpidae) Tropische Natuur. 23:64-70.

1936 - Studies in oriental Mecoptera 1. The genus Leptopanorpa in Malaysia. Treubia. $15: 271-320$.

LinnaEus, C.

1758 - Systemae naturae, sive regna tria naturae systematice proposita por classes, ordines, genera et species. 10. Auflaga. Holmiae.

LLOYD, L.C.

1934 - Two species of Mecoptera from Alaska. Pan-Pacif. Ent. 10:119-120.

LONDT, J.G.H.

1970 - A new species of Bittacus from South Africa (Mecoptera : Bittacidae). J. ent. Soc. sth. Afr. 33:53-57.

1972 - The Mecoptera of southern Africa. J. ent. Soc. sth. Afr. 35:313-343.

1977 - Redescriptions of Bittacus montanus Weele, 1909, and Bittacus schoutedeni Esben-Petersen, 1913, with new synonymy and the de cription of a new central African species (Mlecoptera: Bittacidae). J. ent. Soc. sth. Afr. 40:87-98.

1978 - A new South African Bittacus Latreille, 1809 and a key to southern African Bittacidae (Mecoptera). J. ent. Soc. sth. Afr. 41:189-194.

LONDT, J.G.H. \& BYERS, G.W.

1974 - A new neotropical Bittacus with maculate wings (Mecoptera: Bittacidae). J. Kans. ent. Soc. 47:344-348.

MintYNova, O.M.

1954 - Skorpionnitzy (Mecoptera) fauny SSSR I. Trudy zool, Lening. 15:54-66.
1957 - Skorpionnitzy (Mecoptera) fauny SSSR. II Semejstvo Panorpidae. Ent. Obozr. 36:721-747.

1959 - Skorpionnitzy (Mecoptera) fauny SSSR. III. Semejstvo Bittacidae. Ént. Obozr. 38:196-199.

MATSUMURA, S.

1911 - Erster Beitrag zur Insekten-Fauna von Sachalin. J. Coll. Agric. Hokkaido imp. Univ. 4:1-145.

MAYER, $\mathrm{K}$

1938 - Rod Boreus (Panorpata-Boreidae) v Ceskoslovensku. Ent. Listy. 1:129-140.

MACLACHLAN, $R$.

1867 - New genera and species of Neuropterous Insects; and a revision of $\mathrm{Mr} \mathrm{F}$. Waliker's British-Museum catalogue of Neuroptera, part II (1853), as far as the end of the ge. nus Myrmeleon. J. Linn. Soc. 9:230-281.

1869 - Synopsis of the species of Panorpa occuring in Europe and the adjoining countries; with a description of a singular new species from Java. Trans. R. ent. Soc. Lond. 1869:59-70

1871 - Bittacus apterus nov. sp. Entomologist's mon. Mag. 8:100-102.

1872 - Matériaux pour une faune névroptérologique de l'Asie septentrionale. Seconde partie. Non-Odonates Annls. Soc. ent. Belg. 15:47-78.

1875 - A sketch of our present knowledge of the Neuropterous fauna of Japan (excluding Odonata and Trichoptera). Trans. R. ent. Soc. Lond. 1875:167-190.

1877 - On Notiothauma reedi, a remarkable new genus and species of Neuroptera from Chili, pertaining to the family Panorpidae. Trans. R. ent. Soc. Lond, 1877:427-430.

1881 - On two new Panorpidae from western North America. Entomologist's mon. Mag. 18:36-38.

1882 - A new European Panorpa. Entomologist's mon. Mag. 19:130-132.

1887 - Descriptions de plusieurs nouvelles espèces de Panorpides provenant du Japon et de la Sibérie orientale. Mitt schweiz. ent. Ges. 7:400-406.

1893 - The genus Harpobittacus Gerstaecker. Ent. Nachr. 20:316-317

1894 - On two small collections of Neuroptera from Ta-chien-lu, in the province of Szechuen, Western China, on the frontier of Thibet. Ann. Mag. nat. Hist. (Ser. 6). 13:421-436.

MIYAKÉ, T.

1908 - A list of Panorpidae of Japan, with descrip. tions of ten new species. Bull. Coll. Agric Tokyo imp. Univ. 2:1-12. 
1910 - A further contribution towards the know. ledge of the Panorpidae of Japan. J. Coll. Agric. Tokyo imp. Univ. 2:183-205.

1911 - A synonymic list of the Panorpidae of Ja. pan, with corrections to my former paper, and description of a new species. Entomologist. 44:90-94.

1913 - Studies on the Mecoptera of Japan. J. Coll. Agric. Tokyo imp. Univ. 4:265-400.

MORGANTE, J.S.

1967 - Duas novas espécies de Bittacus Latreille 1807 (Mecoptera Bittacidae), Papéis Dep. Zool. S. Paulo. 20:55-58.

MÜLLER, O.F.

1766 - Manipulus insectorum Taruinensium a Carolo Allionio editus. Miscellania Taruinensis. $3: 185-198$.

NAGLER, C.

1968 - Les Panorpides de la collection du Musée Grigore Antipa-Bucarest. Trav. Mus. Hist. nat. Gr. Antipa. 8:813-820.

1970 - Specii noi de Panorpidae (Ord. Mecoptera). Revta Muzeelor. 7:54-57.

Navís, L.

1908 - Neurópteros nuevos. Mems R. Acad. Cienc. Artes Barcelona, 6:401-423

1909 - Panorpides nouveaux du Japon. Russk. ent. Obozr. 9:273-277.

1910 - Description d'une nouvelle espèce de $\mathrm{Pa}$ norpide (Neur.). Dt. ent. Z. 1910:288-289.

1911 - Névroptères nouveaux de l'extreme Orient. Russk. ent. Obozr. 11:111-117

1912 - Une Panorpide nouvelle de la faune russe (Neuroptera). Russk. ent. Obozr. 12:356-357.

1913a- Neurópteros nuevos de América. Brotéria, 11:45-53.

1913b- Névroptères du Japon recucillis par M. Edme Gallois. Bull. Mus, natn. Hist, nat. 19:441-451.

$1913 c$ - Neuroptera asiatica. I. serie. Russk. ent Obozr. 13:271-284.

1914 - Insectes Névroptères I. Planipennia et Mecoptera. Voyage de $\mathrm{Ch}$. Alluaud et R. Jeannel en Afrique orientale (1911-1912). Résultats scientifiques. 3-52.

1915 - Neurópteros nuevos o poco conocidos (Cuarta serie). Mems R. Acad, Cienc. Artes Barcelona. 11:373-398.

1925 - Algunos insectos del Museo de Paris. 2.A sérle Brotéria. 22:75-83.

1926a - Algunos insèctos del Brasil. Brotéria. 23:5-15. 1926b- Trichoptera, Megaloptera und Neuroptera aus dem Deutsch. Entomolog. Institut (Berlin-Dahlem). II. Serie. Ent. Mitt. 15:57-63.

1926c - Algunos insectos del Museo de Paris. 3.a Serie. Brotéria, 23:95-115.

1926d- Entomologische Ergebnisse der schwedischen Kamtchatka-Expedition 1920-1922. 5. Neuroptera et Mecoptera. Ark. Zool $18 B(2): 1-3$,

1926e- Insectos neotropicos 2." serie. Revta. chil. Hist. nat. 30:326-336.

1927 - Comunicaciones entomológicas 10. Mìs excursiones científicas en 1927. Revta Acad. Cienc. exact. fís-quim. nat. Zaragoza 11:79-137.

1928a - Insectos neotropicos 4." Serie. Revta. chil Hist. nat. 32:106-128.

1928b- Comunicaciones entomológicas 10. Insectos de la India. Revta Acad. Cienc. exact. fis-quim. nat. Zaragoza. 12:177-197.

1929 - Insectos exóticos neurópteros y afines del Museo civico de Génova. Annali Mus. civico Storia nat. Giacomo oria. 53:354-389.

1930a- Névroptèros et insectes voisins - Chine et pays environnants (Première série). Notes Ent. chin. 1(6):1-12.

1930b- Insectes du Congo Belge (Série IV). Revue Zool. Bot. afr. 19:305-336.

1930c - Insecta nova. Séries XV. Neuroptera. Memorie Accad, pont. Nuovi Lincei. (Serie 2). 14:409-418.

1930d-Insecta orientalia. VIII Series. Memorie Accad. pont. Nuovi Lincei. (Série 2). 14:419-434.

1931a- Decadas de insectos nuevos. Revta R. Acad. Cienc, exact. fis. nat. Madr. 26:60-86.

1931b- Insectos del Museo de Paris. 7.' Serie. Brotéria. 27:101-113.

1932a - Alcuni insetti del Museo di Zoologia della R. Università di Torino. Boll. Musei Zool. Anat. comp. R. Univ. Torino. 42(3):1-38.

1932b- Décadas de insectos nuevos. Brotéria. 1:62-82.

1933a - Insecta orientalia. Memorie Accad. pont. Nuovi Lincei (Ser. 2). 17:75-108.

1933b-Insectes du Congo Belge. Série VIII. Revue Zool. Bot. afr. 23:308-318.

1934 - Décadas de insectos nuevos. Brotéria. 3:15-24 .

1935a - Névroptères et insectes voisins - Chine et pays environantes (Huitième série). No. tes Ent. chin. 2:85-203. 


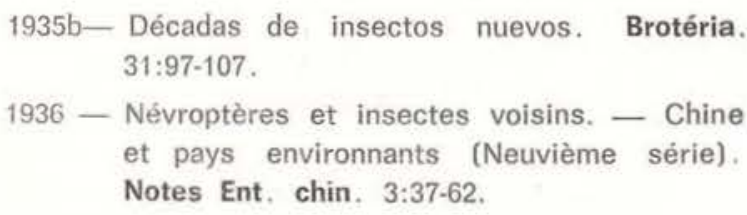

NeEdham, J.G.

1909 - Notes on the Neuroptera in the collection of the Indian Museum. Rec. Indian Mus. $3: 185-210$.

Newman, E.

1838 - Entomological notes. Entomological Mag 5:168-181.

1850 - Description of a new species of Panorpa from New Holland. Proc. R. ent. Soc. Lond. 1850:14.

Окамото, Н

1925 - The Mecoptera of Corea. Bull, agric. Exp. Stn. Chosen. 2:17-24.

PACKARD, A.S.

1870 - New and rare American Neuroptera, Thysanura and Myriapoda. Proc. Boston Soc. nat. Hist. 13:405-411.

PANZER, G.W.

1796 - Faunae insectorum Germaniae initia oder Deutschlands Insecten. Nürnberg.

PENNY, N.D.

1969 - A new species of Bittacus (Mecoptera : Bittacidae) from India. Oriental Insects. 3:161-164

1977a - A systematic study of the family Boreidae (Mecoptera). Kans, Univ. Sci. Bull. 51: 141-217.

1977b- Two new species of Bittacidae (Mecoptera) from the Amazon Basin. Acta Amazônica. $7: 423-427$.

PLIGINSKY, V

1914 - Novye vidy roda Boreus Latr. iz' Kryma (Neuroptera, Panorpidae). Russk. ent. Obozr. 14:363-367.

1930 - Novye vidy roda Boreus Latr. (Neuroptera, Panorpidae). Russk. ent. Obozr. 24:230-231.

Ponomarenko, A.G. \& RASNITSYN, A.P.

1974 - New mesozoic and coenozoic Protomecop. tera (russ.). Paleontol, Zhurnal, Moskva. 4:59-73.

RADERMACHER,

1779 - Verh. batav. Genoot. Kunst. Wet. 1:100.

RAMBUR, M.P.

1842 - Histoire naturelle des insectes. Névroptères, 1-534. Paris

RIEK, $=\mathrm{E}$

1954 - The Australian Mecoptera or scorpion-flies Austr J. Zool. 2:143-168.
1973 - A revision of Australian scorpion flies of the family Choristidae (Mecoptera). J. Austr. ent. Soc. 12:103-112.

RUST, M.K. \& BYERS, G.W.

1976 - The Mecoptera of India and adjacent regions. Kans. Univ. Sci. Bull. 51:19-90.

SAY, T.

1823 - Descriptions of insects belonging to the order Neuroptera Linn. Latr., collected by the expedition authorized by J.-C. Calhoun etc., under the command of major S. $\cdot \mathrm{H}$. Long. Godman's Western Quarterly Reporter. 2:160-165.

Schlee, H.B. \& Schlee, D.

1976 - Bibliographie der rezenten und fossilen Me. coptera (Insecta). Stuttgarter Beitr. Na. turk., Ser. A, Nr. 282: 1-76.

SCHRANK, F, VON P.

1776 - Beyträge zur Naturgeschichte. 1-137. Leipzig.

SELYS-LONGCHAMPS, E.

1868 - Observations sur les Névroptères de Port Denison, reçu par M. Weyers. Annls. Soc. ent. Belg. 11:76-78.

SMTTHERS, C.N .

1959 - A list of the Mecoptera of southern Rhodesia with the description of a new species and key. Proc. R. ent. Soc. Lond. (ser. B). 28:180-184.

1960 - A new species and additional records of Mecoptera from Southern Rodhesia. J. ent. Soc. sth. Afr. 23:45-49.

1973 - New species and records of Australian Bittacidae (Mecoptera). J. Austr. ent. Soc. 12:296-300.

SNODGRASS, R,E.

1927 - Morphology and mechanism of the insect thorax. Smithson. misc. Collns. 80:1-108.

Souza Lopes, H, de \& Mangabeira, O.

1942 - Sobre algumas espécies brasileiras do gênero "Bittacus" Latr., 1807, com a descriçāo de três espécies novas (Panorpatae, Bittacidae). Revta. brasil. Biol. 2:331-341.

SWEDERUS, N.

1787 - Fortsättning af beskrifningen pa 50 nya species af insekter. Kongl. Svenska Vetens. kaps-Acad. nya Handl. 8:276-290.

TARBINSKY, S.P

1962 - O nakhozhdenii lednichnika, Boreus sp. n. (Mecoptera, Boreidae) $v$ predgor'yakh Kirgizskovo Ala-Too. Sbornik entomologische. skikh. Rabot (Akad. Nauk Kirgizsk S.S.R.). 1:131-136. 
TILLYARD, R.J.

1917 - Studies in Australian Mecoptera n. 1. The new family Nannochoristidae, with descriptions of a new genus and four new species: and an appendix descriptive of a new genus and species from New Zealand. Proc. linn. Soc. N.S.W. 42:284-301.

TJEDER, B.

1936 - Schwedisch-chinesische wissenschaftliche Expedition nach den nordwestlichen Provinzen Chinas, unter Leitung von Dr. Sven Hedin und Prof. Su Ping-chang. Insekten gesammelt vom schwedischen Arzt der expedition Dr. David Hummel 1927-1930. Ark. Zool. 27A:1-14.

1951 - Mecopteren aus Fukien. Bonner zool Beitr. 1:286-290.

1956a - Zwei neve ost-asiatische Bittacus-arten (Mecoptera : Bittacidae). Beitr. Ent. 6:45-53.

1956b- Mecoptera. The scorpion flies of Southern Africa. In. Hanstrom, B. et al. (ed): South African animal Life. Results of the Lund University expedition in 1950-1951. Bd. 3:344-390.

1974 - Bittacidae from Burma, collected by R. Malaise (Mecoptera). Ent. Tidskr. 94:188-193.

UHLER, P.R.

1858 - Descriptions of new species of neuropterous insects, collected by the North Pacific exploring expedition under Capt. John Rodgers. Proc. Acad. nat. Sci. Philad. 1858:29-31.

WALKER, F.

1853 - List of the specimens of Neuropterous insects in the collection of the British Museum. Part II (Sialidae-Nemopterides). Pp. 193-476.

WARD, P.H.

1978 - The taxonomy and biology of Panorpa lacedaemonia Lauterbach (Mecoptera, Panorpidae). Entomologist's Gazette. 29:79-98.

WEBB, D.W.

1974 - New species of Panorpa (Mecoptera: Panorpidae). Ent. News. 85:171-173.

WEELE, H.W. VAN DER

1909 - Mecoptera and Planipennia of Insulinde. With biological notes from Edw. Jacobson. Notes Leyden Mus. 31:1-100.
1910 - Neuroptera. 2. Planipennia et Panorpata. In: "Sjostedts Kilimandjaro-Maru Expedition". Bd. 2, Abteil. 13:1-26.

WESTWOOD, J.O.

1840 - A monograph of the genus Panorpa, together with descriptions of species of various allied genera. Ann. Mag. nat. Hist. 8:298-300

1846 - Monograph of the genus Panorpa, with descriptions of some species belonging to other allied genera. Trans. R. ent. Soc. Lond. 4:184-196.

WILLMANN, R.

1973 - Eine neue Panorpa-Art aus Kleinasien: Panorpa aspoecki n. sp. (Mecoptera, Panorpidae). Ent. Z. 83:97-101.

1974 - Panorpa tatvana n. sp. (Mecoptera). Ent. Mitt. zool. Mus. Hamburg. 4:465-469.

1975 - Die Gattung Panorpa (Insecta, Mecoptera) in der Türkei. Annln. naturh. Mus. Wien. 79:543-564.

1976a- Zwei neue Neopanorpa-Arten aus Vietnam (Mecoptera). Annis hist. nat. Mus, natn. hung. 68:65-68.

1976b- Ein neue europäische Panorpa (Mecoptera, Panorpidae). Entomol. Zeitschr. 86:60-64

$1976 \mathrm{c}$ - Zur Kenntnis der italienischen Mecoptera (Insecta). Boll. Mus. Civ. Stor. Nat., Verona. 3:157-177.

1977 - Die subspezifische Gliederung von Panorpa germanica L. auf dem Balkan (Mecoptera: Panorpidae). Entomologica Scandinavica. 8:13-28.

1978 - Bemerkungen zu anatolischen Mecopteren. Ann. Naturhist. Mus. Wien. 81:517-523.

WoOD, H.G.

1933 - Notes on the South African Bittacidae (Mecoptera). Ann. Mag. nat. Hist. (Ser. 10). 12:508-531,

YIE, S.T.

1951 - The biology of Formosa Panorpidae and morphology of eleven species of their immature stages. Mem. Coll. Agric. natn. Taiwan Univ. 2(4):1-111.

(Aceito para publicação em 6/11/78). 